Artículo

\title{
El crecimiento de Agave angustifolia Haw. con relación a la condición nutrimental
}

\author{
Suzel del Carmen Ríos-Ramírez ${ }^{1}$ \\ José Raymundo Enríquez-del Valle ${ }^{2 \S}$ \\ Gerardo Rodríguez-Ortiz ${ }^{2}$ \\ Judith Ruíz-Luna ${ }^{2}$ \\ Vicente Arturo Velasco-Velasco ${ }^{2}$ \\ ${ }^{1}$ TecNM-Instituto Tecnológico Superior de Teposcolula. San Pedro y San Pablo Teposcolula, Oaxaca, \\ México. CP. 69500. (suzelriosramirez@gmail.com). ${ }^{2}$ TecNM-Instituto Tecnológico del Valle de Oaxaca. \\ Ex Hacienda de Nazareno, Xoxocotlán, Oaxaca, México. CP. 71230. Tel. 9515170444. \\ (gerardo.rodriguez@voaxaca.tecnm.mx; judith.rl@voaxaca.tecnm.mx; vicente.vv@voaxaca.tecnm.mx). \\ ${ }^{\S}$ Autor para correspondencia: jose.ev@voaxaca.tecnm.mx.
}

\section{Resumen}

Las plantas de Agave angustifolia Haw., que se obtienen mediante propagación asexual (bulbilos de inflorescencia e hijuelos de rizoma) se establecen en vivero en donde se les proporciona manejo agronómico que se considera debe incluir el abastecimiento de nutrimentos, para lograr plantas de calidad en sanidad y vigor que se establezcan con éxito en campo. La investigación se realizó de enero 2017 a agosto de 2018, en el vivero del Instituto Tecnológico del Valle de Oaxaca, ubicado en la Exhacienda de Nazareno, Xoxocotlán, Oaxaca, México, el objetivo fue evaluar los contenidos nutrimentales en tejidos foliares y el tamaño de las plantas de Agave angustifolia, en condiciones de vivero, fertirrigadas a diferentes concentraciones de nutrimentos. Cuatro grupos de plantas que estuvieron en suelo de textura franco-arenosa, durante siete meses se les aplicó diferentes tipos de riego: agua o solución nutritiva (SN) al 50, 75 y 100\% de la formulación Steiner, transcurrido ese tiempo se evaluó su crecimiento. En muestras de hojas se analizaron los contenidos nutrimentales, y las plantas a las que se aplicó Agua, SN-50\%, SN-75\% y SN-100\%, tuvieron en mg kg-1 de materia seca foliar: 7 225, 10 641, 12575 y 14957 de N; 3 017, 5018,5297 y 6719 de P; 7827 19 604, 16220.1 y $18847 \mathrm{de} \mathrm{Mg}$, acumularon, 282.74, 340.11, 431.98 y $453.05 \mathrm{~g}$ de materia seca foliar, así como 75.01, 77.21, 95.92 y 103.14 g de materia seca en la raíz, respectivamente.

Palabras clave: agave, fertirriego, nutrimentos.

Recibido: junio de 2021

Aceptado: agosto de 2021 


\section{Introducción}

En el estado de Oaxaca, México, el Agave angustifolia se aprovecha como materia prima para la elaboración de mezcal. Es una planta con características morfológicas de suculencia, que forma una gran roseta de 1.5 a $2 \mathrm{~m}$ de altura y 1.5 a $2 \mathrm{~m}$ de diámetro, de hojas rígidas, con espinas marginales y apicales; desarrolla un escapo floral que alcanza hasta $6 \mathrm{~m}$ de altura. El tallo de la roseta, denominada piña, que en promedio su peso fresco es de $80 \mathrm{~kg}$ contiene $21.16 \%$ de azúcares reductores totales del peso fresco (González-González et al., 2007; Cruz et al., 2013), su raíz es superficial, crece en terrenos con pendientes moderadas a pronunciadas, suelos pedregosos.

La especie soporta condiciones ambientales, tales, como déficit hídrico, alta temperatura, suelos moderadamente salinos y de fertilidad baja, que limitan el crecimiento y productividad de otras plantas de cultivo (Andrade et al., 2007; García, 2007; Enríquez-del-Valle, 2008). Esta especie presenta fotosíntesis de tipo metabolismo ácido de las crasuláceas, MAC (Matiz et al., 2013) y que permite obtener ganancias netas de carbono con pérdidas mínimas de agua (Winter y Smith, 1996).

Al ser una planta alcoholígena de interés económico para la elaboración de mezcal, se necesita material vegetal para establecer en campo. Aun cuando esta especie produce semillas viables, las plantaciones se establecen con plantas que se obtienen mediante propagación asexual, principalmente hijuelos de rizoma y bulbilos de inflorescencia, y en una pequeña proporción se han establecido plantas obtenidas mediante cultivo de tejidos vegetales (Arizaga y Ezcurra, 2002; Enríquez-del Valle, 2008).

Las plantas que se obtienen de manera asexual se mantienen durante seis a 18 meses en vivero, para obtener de 30-50 cm de altura, conveniente para establecerlas en campo y durante este periodo es necesario dar un manejo agronómico, etapa en la que se considera conveniente la sanidad y el abastecimiento de nutrimentos para lograr plantas de calidad y vigor que se establezcan con éxito en campo. Cuando las plantas se extraen del suelo de vivero para transferirlas a campo, se les podan las raíces y en ocasiones se sumergen durante unos minutos en solución fungicida, para colocarlas posteriormente en un sitio ventilado a la sombra durante dos días a una semana para que sus tejidos pierdan ligeramente humedad, lo cual según los viveristas promueve que la planta se establezca con éxito en el campo.

Las plantaciones se establecen durante los primeros meses de la temporada de lluvias, para asegurar que las plantas formen nuevas raíces para establecerse en suelo y reinicien crecimiento (Díaz et al., 2011). Las plantas en general dependen de la energía de la radiación solar captada mediante el proceso fotosintético para la fijación de carbono y la síntesis de compuestos estructurales y metabólicos. Pero la eficiencia de la planta para captar radiación solar, fijar carbono y acumular materia seca depende de su condición fisiológica que está relacionada con la nutrimental.

En A. tequilana se han realizado estudios de fertilidad de suelos, que concluyen la necesidad de aplicar fertilizantes para aumentar los rendimientos de los cultivos (Álvarez-Sánchez et al., 2010). A. angustifolia en condiciones de campo (Martínez-Ramírez y Bautista-Sánchez, 2013), así como en plantas micropropagadas de A. americana var. oaxacensis (Enríquez-del-Valle et al., 2013) en invernadero y A. potatorum (Enríquez-del Valle et al., 2016) en condiciones de vivero, muestran el efecto favorable del abastecimiento nutrimental en el crecimiento de las plantas. Son 17 los 
nutrimentos esenciales que se clasifican en macronutrimentos (nitrógeno, N, fósforo, $\mathrm{P}$, potasio $\mathrm{K}$, calcio, $\mathrm{Ca}$, magnesio, $\mathrm{Mg}$ y azufre, $\mathrm{S}$ ) y micro nutrimentos (hierro, $\mathrm{Fe}$, cobre, $\mathrm{Cu}$, manganeso, $\mathrm{Mn}$, zinc, Zn, boro, B y molibdeno, Mo), (Vitosh et al., 1994; Grusak, 2001; Zúñiga, 2013).

De todos los elementos esenciales para la planta, el carbono $(\mathrm{C})$, el hidrógeno $(\mathrm{H})$ y el oxígeno $(\mathrm{O})$ constituyen cerca de $90 \%$ del peso seco de una planta (Medina, 1999). Dentro de la planta, estos elementos se usan para la síntesis de carbohidratos, mediante la fotosíntesis, la cual se realiza en los cloroplastos de las células, utilizando la luz como fuente de energía. Los 14 elementos restantes solo representan alrededor de $10 \%$ del peso seco de una planta (Rodríguez y Flores, 2004).

En trabajos previos de desarrollo en invernadero-vivero de A. angustifolia, se ha demostrado la conveniencia de suministrar fertirriego la cual tiene efectos positivos en el desarrollo de las plantas, en características tales como número de hojas, altura de la planta, diámetro de tallo, pesos frescos y secos (Enríquez-del-Valle et al., 2009). Pero se requiere más información para agave que muestre la relación entre abastecimiento nutrimental, contenido de nutrimentos en los tejidos de la planta y el crecimiento de ésta. Por lo que el objetivo de este trabajo fue: Evaluar plantas de A angustifolia, que en condiciones de vivero se fertirrigaron a diferentes concentraciones de nutrimentos, el tamaño que alcanzaron en relación con los contenidos nutrimentales de sus tejidos foliares.

\section{Materiales y métodos}

\section{Material experimental}

Este trabajo es continuación del procedimiento experimental, en que se reportaron datos de crecimiento de las plantas, por Ríos-Ramírez et al. (2018). El material vegetal consistió en 27 plantas de 18 meses de edad de A. angustifolia obtenidas a partir de bulbilos de inflorescencia. Las cuales se encontraban en condiciones de vivero, establecidas individualmente en macetas de $4 \mathrm{dm}^{3}$ con suelo de textura franco-arenosa. Se separaron en cuatro grupos, los que recibieron dos veces a la semana durante siete meses, diversos tipos de riego (tratamientos): 1) solo agua; o riego con solución nutritiva (SN) en diferentes diluciones de la formulación de Steiner (1984), 2) SN-50\%; 3) $\mathrm{SN}-75 \%$; y 4) $\mathrm{SN}-100 \%$.

La SN-100\% contiene en mg L ${ }^{-1}: 166.42$ N, 30.68 P, 276.44 K, 182.34 Ca, 49.09 Mg, 111.15 S, 1.25 Fe, 0.21 Mn, 0.025 Zn, 0.076 B, 0.005 Cu y 0.021 Mo. También, a todos los grupos se le aplicó dos veces a la semana aspersiones con fungicida agrícola $\mathrm{N}$-triclorometiltio-4-ciclohexeno1,2-dicarboximida de la Marca Bayer ${ }^{\circledR}$ y antibiótico sistémico, terramicina agrícola al 5\% Oxitetraciclina, de la marca Zoetis ${ }^{\circledR}$. Transcurridos los siete meses en las condiciones citadas, las plantas se cosecharon y se cuantificó su altura, número de hojas, área foliar $\left(\mathrm{cm}^{2}\right)$ que se determinó con un scanner Laser Área Meter (CID Bio-Science, Copyrigh ${ }^{\circledR} 2016$ CID Bio-Sciencie, Inc. Camas, WA EE. UU.), el volumen foliar y de raíz $\left(\mathrm{cm}^{3}\right)$ al sumergirlos en un volumen conocido de agua en una probeta graduada.

El peso fresco $(\mathrm{g})$ foliar y de raíz se midió en una balanza de barra triple (IROSA ${ }^{\circledR} 700$ PPW, México) con precisión de $0.1 \mathrm{~g}$. Las hojas y raíces se colocaron por separado en bolsas de papel debidamente etiquetadas, para colocarlas durante dos semanas a una temperatura de $65{ }^{\circ} \mathrm{C}$, en una estufa de convección (Felisa ${ }^{\circledR}$ Fabricantes Feligneo, SA de CV, México). Transcurrido este tiempo nuevamente en la balanza de barra triple se pesaron el material seco de raíz y foliar. 


\section{Determinación de nutrimentos en tejidos foliares}

Para analizar los contenidos nutrimentales, las hojas secas de Agave se pulverizaron en un molino (Apex Construction Ltd V110), de las cuales se pesaron $0.5 \mathrm{~g}$ de muestra por duplicado en una balanza analítica (Sartorius CP2245), estas muestras fueron sometidas a digestión húmeda con una mezcla de $\mathrm{HNO}_{3}: \mathrm{HClO}_{4}$ (relación 2:1) en un digestor Kjeldahl marca SEV modelo DIK-20.

La determinación de nitrógeno $(\mathrm{N})$ y carbono $(\mathrm{C})$ se realizó por el método de combustión, en un Analizador orgánico elemental (Mod: Perkin Elmer Serie II CHNS/0 analyzer 2400), el fósforo (P) se evaluó por el método de Vanadato-Molibdato-Amarillo en un espectrofotómetro de luz ultravioleta visible (UV-Vis GBC Cintra 10) a una longitud de onda de $470 \mathrm{~nm}$, los elementos potasio $(\mathrm{K})$, magnesio $(\mathrm{Mg})$, sodio $(\mathrm{Na})$, cobre $(\mathrm{Cu})$, fierro $(\mathrm{Fe})$, manganeso $(\mathrm{Mn})$ y zinc $(\mathrm{Zn})$, se determinaron por Espectrofotometría de Absorción Atómica, en un espectrofotómetro de absorción atómica (Thermo Scientific, modelo ICE 3000 series AA) con llama aire-acetileno por aspiración directa; y los sulfatos $\left(\mathrm{SO}_{4}\right)$ por el método turbidimétrico, en un espectrofotómetro de luz ultravioleta visible (UV-Vis GBC Cintra 10) a una longitud de onda de $340 \mathrm{~nm}$.

\section{Diseño experimental y análisis de datos}

El experimento se estableció de acuerdo con un diseño completamente al azar. La unidad experimental fue una planta y con diferente cantidad de repeticiones por tratamiento (de cuatro a 11). En los datos de cada variable se analizó la homogeneidad de varianza mediante la prueba de Bartlet y la prueba de normalidad Shapiro-Wilk. Las variables $\mathrm{N}, \mathrm{Na}, \mathrm{K}, \mathrm{Zn}, \mathrm{Fe}, \mathrm{Cu}$ se transformaron a $\log 10(\mathrm{x})$, para el cumplimiento de los supuestos de homogeneidad $\mathrm{y}$ normalidad. Los datos se sometieron a análisis de varianza, comparaciones de medias (Duncan, 0.05), correlaciones (0.05). Se realizaron análisis de correlación entre el peso seco foliar y los contenidos de nutrimentos en las hojas, así como entre las cantidades de los diversos nutrimentos. Para las rutinas de análisis estadísticos se usó el programa computacional SAS ${ }^{\circledR}$ Statistical Analysis System, version 9.0.

\section{Resultados y discusión}

Los contenidos nutrimentales encontrados en tejidos foliares de las plantas de A. angustifolia, estuvieron influenciados por las concentraciones crecientes de fertirriego que recibieron durante siete meses en vivero. Los análisis de varianza (Cuadro 1) muestran que los niveles de abastecimiento nutrimental tuvieron efectos diferentes significativos $(p \leq 0.05)$ en los contenidos de $\mathrm{N}, \mathrm{Mg}, \mathrm{Zn}$ y no significativo para el $\mathrm{K}, \mathrm{SO}_{4}, \mathrm{P}, \mathrm{Na}, \mathrm{Fe}, \mathrm{Cu}$, así como en la materia seca foliar acumulada.

La cantidad de materia seca foliar y materia seca de raíz que las plantas acumularon, también fueron en relación positiva a la cantidad de nutrimentos que se les suministró. De tal manera que las plantas irrigadas con solo agua y las plantas fertirrigadas con la SN-100\%, acumularon 282.7 y $453.05 \mathrm{~g}$ de materia seca foliar, así como 75 y 103.1 g de materia seca en la raíz, que en cada caso fueron magnitudes significativamente (Duncan, 0.05) diferentes (Cuadro 2). 
Cuadro 1. Resumen de análisis de varianza de materia seca y contenidos nutrimentales en tejidos foliares de plantas de Agave angustifolia que en vivero se fertirrigaron a diferentes concentraciones de nutrimentos.

\begin{tabular}{cccccccc}
\hline & & \multicolumn{5}{c}{ Cuadrados medios y significancia } \\
\cline { 3 - 8 } FV & Gl & $\mathrm{P}$ & $\mathrm{C}$ & $\mathrm{N}$ & $\mathrm{Na}$ & $\mathrm{Mg}$ & $\mathrm{K}$ \\
\hline Trat & 3 & $14847174.6^{\mathrm{ns}}$ & $489539571^{\mathrm{ns}}$ & $68167267.3^{*}$ & $1.60107727^{\mathrm{ns}}$ & $19863894^{*}$ & $0.33048079^{\mathrm{ns}}$ \\
Err & 24 & 12454715.2 & 668481315 & 10267603.5 & 0.57726192 & 61911784 & 0.12158501 \\
Tot & 27 & & & & & & \\
Fv & Gl & Zn & $\mathrm{Fe}$ & $\mathrm{SO}_{4}$ & $\mathrm{Cu}$ & $\mathrm{MSF}$ & $\mathrm{MSR}$ \\
Trat & 3 & $0.27210426^{*}$ & $0.20388993^{\mathrm{ns}}$ & $36743275.7^{\mathrm{ns}}$ & $0.1886378^{\mathrm{ns}}$ & $2.67^{\mathrm{ns}}$ & $1508.2^{\mathrm{ns}}$ \\
Err & 24 & 0.08453928 & 0.4057517 & 21039730.1 & 0.35015818 & 0.96 & 1869.2 \\
Tot & 27 & & & & & & \\
\hline
\end{tabular}

$\mathrm{Fv}=$ fuentes de variación; $\mathrm{Gl}=$ grados de libertad; Trat= tratamiento; Err= error; Tot= total; $\mathrm{P}=$ fósforo; $\mathrm{C}=\mathrm{carbono}$; $\mathrm{N}=$ nitrógeno; $\mathrm{Na}=$ sodio; $\mathrm{Mg}=$ magnesio; $\mathrm{K}=$ potasio $\mathrm{Zn}=$ zinc $; \mathrm{Fe}=$ hierro $\mathrm{SO}_{4}=$ sulfatos; $\mathrm{Cu}=\mathrm{cobre} ; \mathrm{MSF}=$ materia seca foliar; $\mathrm{MSR}=$ materia seca de raíz; $\mathrm{ns}=$ valores no significativos $(p>0.05) ;{ }^{*}=$ valores con efectos significativos $(p \leq 0.05){ }^{* *}=$ valores con efectos altamente significativos $(p \leq 0.01)$.

Cuadro 2. Materia seca foliar (g) y de la raíz (g), y los contenidos nutrimentales (mg $\mathrm{kg}^{-1}$ ) en tejidos foliares de plantas de Agave angustifolia que en vivero se fertirrigaron a diferentes concentraciones de nutrimentos.

\begin{tabular}{ccccc}
\hline \multirow{2}{*}{ Variables } & \multicolumn{4}{c}{ Tratamiento de tipo de riego } \\
\cline { 2 - 5 } & Agua & SN50\% & SN75\% & SN100\% \\
\hline MSF & $282.74 \pm 0.6 \mathrm{~b}$ & $340.11 \pm 1 \mathrm{ab}$ & $431.98 \pm 0.7 \mathrm{ab}$ & $453.05 \pm 121.5 \mathrm{a}$ \\
$\mathrm{MSR}$ & $75.01 \pm 32.6 \mathrm{a}$ & $77.21 \pm 48 \mathrm{a}$ & $95.92 \pm 25.7 \mathrm{a}$ & $103.14 \pm 53.4 \mathrm{a}$ \\
$\mathrm{P}$ & $3017 \pm 3024.4 \mathrm{a}$ & $5018 \pm 4009.5 \mathrm{a}$ & $5297 \pm 2783 \mathrm{a}$ & $6719 \pm 537.9 \mathrm{a}$ \\
$\mathrm{C}$ & $413508 \pm 250382.1 \mathrm{a}$ & $403786 \pm 423534.2 \mathrm{a}$ & $395363 \pm 217209.9 \mathrm{a}$ & $416057 \pm 55592.8 \mathrm{a}$ \\
$\mathrm{N}$ & $7225 \pm 5773.8 \mathrm{c}$ & $10641 \pm 16475.7 \mathrm{bc}$ & $12575 \pm 9162.3 \mathrm{ab}$ & $14957 \pm 2381.6 \mathrm{a}$ \\
$\mathrm{Mg}$ & $7827 \pm 4950.5 \mathrm{~b}$ & $19604 \pm 9389.9 \mathrm{a}$ & $16220.1 \pm 4670.3 \mathrm{ab}$ & $18847 \pm 992.4 \mathrm{a}$ \\
$\mathrm{K}$ & $18057 \pm 16352.4 \mathrm{a}$ & $45048 \pm 16475.7 \mathrm{a}$ & $42522 \pm 9956.9 \mathrm{a}$ & $45943 \pm 3094.2 \mathrm{a}$ \\
$\mathrm{Na}$ & $153.2 \pm 265 \mathrm{a}$ & $892.7 \pm 16475.7 \mathrm{a}$ & $543.2 \pm 463.9 \mathrm{a}$ & $779 \pm 124.9 \mathrm{a}$ \\
$\mathrm{Zn}$ & $12.88 \pm 11.5 \mathrm{a}$ & $26.89 \pm 15.4 \mathrm{a}$ & $29.23 \pm 26.8 \mathrm{a}$ & $33.85 \pm 0.0004 \mathrm{a}$ \\
$\mathrm{Fe}$ & $23.55 \pm 23.3 \mathrm{a}$ & $65.32 \pm 56.8 \mathrm{a}$ & $67.37 \pm 53.6 \mathrm{a}$ & $55.02 \pm 0.0009 \mathrm{a}$ \\
$\mathrm{Cu}$ & $14.83 \pm 12.1 \mathrm{a}$ & $36.63 \pm 30.5 \mathrm{a}$ & $46.14 \pm 42.7 \mathrm{a}$ & $30.21 \pm 0.0004 \mathrm{a}$ \\
$\mathrm{SO}_{4}$ & $1371 \pm 1957.8 \mathrm{a}$ & $6087 \pm 4607 \mathrm{a}$ & $5843 \pm 3934.5 \mathrm{a}$ & $6510 \pm 0.07 \mathrm{a}$ \\
\hline
\end{tabular}

$\mathrm{P}=$ fósforo; $\mathrm{C}=$ carbono; $\mathrm{N}=$ nitrógeno; $\mathrm{Na}=$ sodio; $\mathrm{Mg}=$ magnesio; $\mathrm{K}=$ potasio; $\mathrm{Zn}=$ zinc; $\mathrm{Fe}=$ hierro $\mathrm{SO}_{4}=$ sulfatos; $\mathrm{Cu}=$ cobre; $\mathrm{MSF}=$ materia seca foliar; $\mathrm{MSR}=$ materia seca de raíz. En cada variable en sentido horizontal, medias con la misma letra no son significativamente diferentes (Duncan, 0.05).

De acuerdo con la concentración para cada nutrimento determinado en tejidos foliares de $A$. angustifolia, los macroelementos con mayor concentración fueron $\mathrm{C}, \mathrm{N}, \mathrm{Mg}, \mathrm{K}$ y el microelemento Fe, en comparación al resto de los nutrimentos determinados (Cuadro 2). Las plantas acumularon en sus tejidos foliares más $\mathrm{N}, \mathrm{P}$ y $\mathrm{Zn}$ en respuesta a las cantidades crecientes de nutrimentos en fertirriego, hasta $\mathrm{SN}-100 \%$, de tal manera las plantas irrigadas con agua y las plantas fertirrigadas con SN-100\% tuvieron en mg kg-1 de materia seca: 7225 y 14957 de N, 3017 y 6719 de P, 12.88 y 33.85 de Zn, respectivamente, cantidades que en cada caso fueron significativamente diferentes (Duncan, 0.05). 
En el caso de $\mathrm{Fe}$ y $\mathrm{Cu}$ se observó una tendencia similar, pero la máxima acumulación de estos nutrimentos ocurrió cuando se aplicó hasta SN-75\%. Las plantas irrigadas con solo agua tuvieron en sus tejidos foliares $1371 \mathrm{mg} \mathrm{kg}^{-1}$ de $\mathrm{SO}_{4}$ y $18057 \mathrm{mg} \mathrm{kg}^{-1}$ de $\mathrm{K}$ de materia seca, mientras que en las plantas fertirrigadas se observó una tendencia, no significativa, de tener más $\mathrm{SO}_{4}$ y K (Cuadro 2). Por lo que se puede decir que el abastecimiento creciente de solución nutritiva a las plantas de A. angustifolia durante siete meses acumuló cantidad mayor de nutrimentos en los tejidos foliares de estas plantas, las que mostraron crecimiento mayor.

Los datos de análisis nutrimental de A. angustifolia obtenidos en el presente trabajo concuerdan con los reportados por Cruz et al. (2013) quienes, en plantas adultas de esta especie, provenientes de tres localidades de Oaxaca, México, se les determinó mayor concentración de los macroelementos Mg y Ca; y el microelemento Fe. Silos-Espino et al. (2011) Estudiaron plantas de ocho a 12 años (antes de floración) de A. salmiana Gentry en las que analizaron componentes de valor alimenticio en calidad forrajera y principales minerales ( $\mathrm{Ca}, \mathrm{P}, \mathrm{Mg}, \mathrm{Fe}, \mathrm{Zn}, \mathrm{Cu}, \mathrm{Bo}$ y $\mathrm{Se}$ ) de

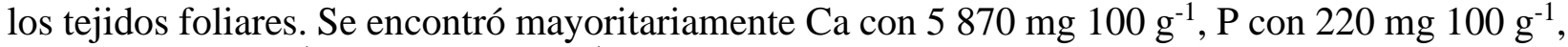

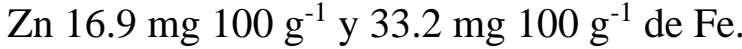

Las plantas fijan el $\mathrm{CO}_{2}$ atmosférico a través del proceso de fotosíntesis y sintetizan compuestos orgánicos (Glime 2017). Posteriormente los productos de la fotosíntesis y las sustancias minerales del suelo son utilizados en los procesos de síntesis de proteínas, lípidos y otros compuestos orgánicos que son constituyentes estructurales y metabólicos de las plantas. Y de la eficiencia de estos procesos resulta la magnitud de biomasa acumulada y el rendimiento económico (GutiérrezRodríguez et al. 2005). Ya que interesa que las plantas en vivero muestren crecimiento vigoroso, se debe dar atención que éstas tengan fotosíntesis neta alta, además de sanidad de la planta, la eliminación de otras especies vegetales competidoras y que las plantas se encuentren en apropiada condición hídrica y nutrimental.

Los análisis nutrimentales de las plantas de A. angustifolia, muestran diferencias entre los diferentes grupos de plantas de acuerdo a la cantidad de fertirriego que recibieron (Agua, SN-50\%, $\mathrm{SN}-75 \%$, SN-100\%) y que tienen relación con los datos reportados por Ríos-Ramírez et al. (2018) que muestran que transcurridos siete meses en vivero, las plantas de A. angustifolia incrementaron su cantidad de hojas, el tamaño de la raíz y de las hojas, evaluadas en área foliar, volumen foliar, peso seco foliar, en relación positiva a la cantidad de nutrimentos que recibieron mediante el riego.

Ya que las plantas que recibieron mayor dosis de fertilización tuvieron mayor concentración de nutrimentos en su materia seca foliar y también fueron las más grandes (Cuadro 2). Por lo que se muestra la conveniencia del fertirriego, ya que este mantiene la disponibilidad de agua y elementos nutritivos en la zona radical a niveles óptimos, para obtener un crecimiento mayor de las plantas y posteriormente la productividad potencial del cultivo (Guzmán, 2004).

En árboles jóvenes de especies forestales que se producen en vivero, también se ha demostrado que la condición nutrimental de la planta tiene efecto en la altura total, el diámetro del tallo, la biomasa aérea y de raíz, características que tienen relación con la capacidad de la planta para superar la condición de estrés cuando se establece en campo y reinicia crecimiento (McDowell et al., 2008). En general las plantas necesitan nutrimentos para la síntesis de sustancias estructurales y metabólicas, que determina su eficiencia fotosintética para la asimilación de $\mathrm{C}$ que constituye entre 40 a 50\% de la materia seca vegetal, también mediante la fotosíntesis la energía de la radiación solar se conserva en compuestos que aportan energía para los procesos de síntesis (Grusak, 2001). 
A mayor concentración de solución nutritiva suministrada a las plantas éstas crecieron más y sus tejidos foliares tuvieron mayor concentración de $\mathrm{N}$ y este elemento $\mathrm{N}$ forma parte de compuestos metabólicamente importantes como aminoácidos, proteínas, pigmentos fotosintéticos, por lo que su abastecimiento tiene una acción directa sobre el incremento de la materia seca (McDonald et al., 1996; Uchida 2000). Los análisis de correlación (Cuadro 3) muestran que los pesos secos foliares tuvieron correlación positiva y significativa con el contenido de $\mathrm{N}(\mathrm{r}=0.678, p \leq 0.01)$, con el fósforo ( $\mathrm{r}=0.743, p \leq 0.01)$, con $\mathrm{Mg}(\mathrm{r}=0.899, p \leq 0.01)$, con $\mathrm{K}(\mathrm{r}=0.83, p \leq 0.01)$, con $\mathrm{Fe}$ $(\mathrm{r}=0.786, p \leq 0.01), \mathrm{Cu}(\mathrm{r}=0.699, p \leq 0.01), \mathrm{SO}_{4}(\mathrm{r}=0.977, p \leq 0.01)$.

Cuadro 3. Correlaciones entre la materia seca foliar (g) y los contenidos nutrimentales ( $\left.\mathrm{mg} \mathrm{kg}^{-1}\right)$ en tejidos foliares de plantas de Agave angustifolia que durante siete meses en vivero se fertirrigaron a diferentes concentraciones de nutrimentos.

\begin{tabular}{|c|c|c|c|c|c|c|c|c|c|c|c|}
\hline & $\mathrm{T}$ & MSF & $\mathrm{P}$ & $\mathrm{C}$ & $\mathrm{N}$ & $\mathrm{Mg}$ & $\mathrm{K}$ & $\mathrm{Mn}$ & $\mathrm{Zn}$ & $\mathrm{Fe}$ & $\mathrm{SO}_{4}$ \\
\hline $\mathrm{T}$ & 1 & $0.43^{*}$ & $0.36^{\mathrm{ns}}$ & $-0.002^{\mathrm{ns}}$ & $0.67^{* *}$ & $0.404^{*}$ & $0.34^{\mathrm{ns}}$ & $0.53^{\mathrm{ns}}$ & $0.39^{*}$ & $0.22^{\mathrm{ns}}$ & $0.37^{*}$ \\
\hline MSF & & 1 & $0.74^{* *}$ & $0.036^{\mathrm{ns}}$ & $0.68^{* *}$ & $0.899^{* *}$ & $0.83^{* *}$ & $-0.11^{\mathrm{ns}}$ & $0.5^{*}$ & $0.79^{* *}$ & $0.98^{* *}$ \\
\hline $\mathrm{P}$ & & & 1 & $-0.192^{\mathrm{ns}}$ & $0.03^{\mathrm{ns}}$ & $0.63^{* *}$ & $0.55^{*}$ & $0.05^{\mathrm{ns}}$ & $0.56^{* *}$ & $0.61^{* *}$ & $0.74^{* *}$ \\
\hline $\mathrm{C}$ & & & & 1 & $0.11^{\mathrm{ns}}$ & $0.002^{\mathrm{ns}}$ & $-0.05^{\mathrm{ns}}$ & $0.94^{\mathrm{ns}}$ & $0.09^{\mathrm{ns}}$ & $-0.06^{\mathrm{ns}}$ & $0.08^{\mathrm{ns}}$ \\
\hline $\mathrm{N}$ & & & & & 1 & $0.845^{* *}$ & $0.72^{* *}$ & $-0.57^{\mathrm{ns}}$ & $0.45^{*}$ & $0.55^{*}$ & $0.87^{* *}$ \\
\hline $\mathrm{Mg}$ & & & & & & 1 & $0.89^{* *}$ & $-0.24^{\mathrm{ns}}$ & $0.62^{*}$ & $0.66^{*}$ & $0.87^{* *}$ \\
\hline $\mathrm{K}$ & & & & & & & 1 & $0.22^{\mathrm{ns}}$ & $0.53^{*}$ & $0.52^{*}$ & $0.82^{* *}$ \\
\hline Mn & & & & & & & & 1 & $0.87^{\mathrm{ns}}$ & $-0.25^{\mathrm{ns}}$ & $-0.08^{\mathrm{ns}}$ \\
\hline $\mathrm{Zn}$ & & & & & & & & & 1 & $0.37^{\mathrm{ns}}$ & $0.45^{*}$ \\
\hline $\mathrm{Fe}$ & & & & & & & & & & 1 & $0.8^{* *}$ \\
\hline $\mathrm{SO}_{4}$ & & & & & & & & & & & 1 \\
\hline
\end{tabular}

$\mathrm{T}=$ dosis de fertilización; $\mathrm{P}=$ fósforo; $\mathrm{C}=$ carbono; $\mathrm{N}=$ nitrógeno; $\mathrm{Mg}=$ magnesio; $\mathrm{K}=$ potasio; $\mathrm{Ca}=$ calcio; $\mathrm{Zn}=$ zinc; $\mathrm{Fe}=$ fierro $\mathrm{SO}_{4}=$ sulfatos; $\mathrm{Cu}=$ cobre; $\mathrm{MSF}=$ materia seca foliar. En cada valor de correlación, $\mathrm{ns}=$ no significativo $(p>$ $0.05) ;{ }^{*}=$ significativo $(p \leq 0.05) .{ }^{* *}=$ altamente significativo $(p \leq 0.01)$.

\section{Conclusiones}

Plantas de Agave angustifolia Haw. Acumularon más materia seca foliar y de raíz, y tuvieron más $\mathrm{N}, \mathrm{P}, \mathrm{K}, \mathrm{Zn}, \mathrm{Fe}, \mathrm{Cu}$ y $\mathrm{SO}_{4}$ que plantas no fertirrigadas y las cantidades se incrementaron en relación positiva a los nutrimentos que recibieron. Las plantas irrigadas con solo agua y las plantas fertirrigadas a 75 o $100 \%$ de nutrimentos de la formulación de Steiner, tuvieron 282.74, 431.98 y $453.05 \mathrm{~g}$ de materia seca foliar, 75.01, 95.92 y $103.14 \mathrm{~g}$ de materia seca en la raíz, sus hojas tuvieron, en mg kg-1 de materia seca: 3 017, 5297 y 6719 de P; 7225,12575 y 14957 de N; $12.88,29.23$ y 33.85 de Zn; 23.55, 67.37 y 55.02 de Fe; 14.83, 46.14 y 30.21 de Cu, respectivamente. En general la materia seca foliar tuvo correlaciones altas positivas, significativas, con los contenidos de N, P, K, Mg, Zn, Fe, Cu, $\mathrm{SO}_{4}$. 


\section{Literatura citada}

Álvarez-Sánchez, M. E.; Velázquez-Mendoza, J.; Maldonado-Torres, R.; Almaguer-Vargas, G. y Solano-Agama, A. L. 2010. Diagnóstico de la fertilidad y requerimiento de cal de suelos cultivados con agave azul (Agave tequilana Weber). Terra Latinoam. 28(3):287-293.

Andrade, J. L. E.; Reyes-García, C.; Ricalde, M. F.; Vargas-Soto, G. y Cervera, J. C. 2007. El metabolismo ácido de las crasuláceas: diversidad, fisiología ambiental y productividad. Boletín de la Sociedad Botánica de México. 81:37-50.

Arizaga, S. and Ezcurra, E. 2002. Propagation mechanisms in Agave macroacantha (Agavaceae), a tropical arid-land succulent rosette. Am. J. Bot. 89(4):32-41.

Cruz, H.; Enríquez-del-Valle, J. R.; Velasco, V. A.; Ruiz, J.; Campos, G. V. y Aquino, D. E. 2013. Nutrimentos y carbohidratos en plantas de Agave angustifolia Haw. y Agave karwinskii Zucc. Rev. Mex. Cienc. Agríc. 6(4):1161-1173.

Díaz, J. G.; Rojas, G.; Him, F. Y.; Hernández, B. N.; Torrealba, E. y Rodríguez, Z. 2011. Efecto de la fertilización nitrogenada sobre el crecimiento en vivero de Cocuy (Agave cocui Trelease). Rev. Facult. Agron. Luz. 28(1):264-272.

Enríquez-Valle, J. R. 2008. La propagación y crecimiento de agaves. Fundación Produce Oaxaca, AC. e Instituto Tecnológico del Valle De Oaxaca. Oaxaca, México. 42 p.

Enríquez-Valle, J. R.; Velasco-Velasco, V. A.; Campos-Ángeles, G. V.; Hernández-Gallardo, E. and Rodríguez-Mendoza, M. N. 2009. Agave angustifolia plants grown with different fertigation doses and organic substrates. Acta Hortic. 843:49-55.

Enríquez-Valle, J. R.; Estrada, A.; Rodríguez, G.; Velasco, V. A. y Campos, G. V. 2013. Sustrato y dosis de fertirriego en la aclimatación de vitroplantas de Agave americana var. oaxacencis. Rev. Facul. Cienc. Agr. Uncuyo. 45(2):341-348.

Enríquez-Valle, J. R.; Alcara-Vásquez, S. E.; Miguel-Luna, M. E. y Manuel-Vásquez, C. 2016. Fertirriego en vivero a plantas de Agave potatorum Zucc. micropropagadas-aclimatizadas. Rev. Mex. Cienc. Agríc. 7(5):1167-1177. http://www.redalyc.org/articulo.oa?id= 263146723016.

García, A. J. 2007. Los agaves de México. Rev. Cienc. 87:14-23.

Glime, J. M. 2017. Photosynthesis: the process. In: Glime J. M. (Ed.) bryophyte ecology. Physiological ecology. Ebook sponsored michigan technological university and the international association of bryologists. USA. 1-11 p.

González-González, L. R.; García-Pérez, M. I. B.; Gutiérrez, L. K. y García, A. 2007. Obtención de azúcares fermentables a partir de inulinasas inmovilizadas por el método del sol-gel. Rev. Cienc. Tecnol. 6(6):106-111.

Grusak, M. A. 2001. Plant macro-and micronutrient minerals. Encyclopedia of life sciences center, Texas, USA. 5 p.

Gutiérrez-Rodríguez, M.; Reynolds, M. P.; Escalante, E. y Larqué-Saavedra, J. A. 2005. Algunas consideraciones en la relación entre fotosíntesis y el rendimiento de grano en trigo. Rev. Cienc. Ergo Sum. 122):149-154.

Guzmán, M. 2004. Población, agua, suelo y fertilizantes: el ferti-riego. In: ferti-riego: tecnologías y programación en agroplasticultura. (Ed.). Programa CYTED Secretaría General. Madrid, España. 5-10 p.

Martínez-Ramírez, S. y Bautista-Sánchez, G. 2013. Adaptabilidad de Agave potatorum Zucc. a las condiciones ambientales y socioeconómicas de río azucena, San Juan Mixtepec, Oaxaca. Temas de Ciencia y Tecnología. 17(50):3-12. 
Martínez-Ramírez, S.; Trinidad-Santos, A.; Bautista-Sánchez, G. y Pedro-Santos, E. C. 2013. Crecimiento de plántulas de dos especies de mezcal en función del tipo de suelo y nivel de fertilización. Rev. Fitot. Mex. 36(4):387-393.

Matiz, A.; Tamaso, M. P.; Yepes, M. A.; Freschi, L. and Helenice, M. 2013. CAM Photosynthesis in bromeliads and agaves: What can we learn from these plants? Creative Commons. 1:91-132.

McDonald, J.; Ericsson, T. and Larsson, C. M. 1996. Plant nutrition, dry mater gain and partitioning at the whole-plant level. J. Exp. Bot. 47:1245-53.

McDowell, N.; Pockman, W. T.; Allen, C. D.; Breshears, D. D.; Cobb, N. and Kolb, T. 2008. Mechanisms of plant survival and mortality during drought: why do some plants survive while others succumb to drought? New Phytologist, Tansley Review. J. Compilation 178(4):719-739.

Medina, A. 1999. Manejo de la nutrición en el rosal después de la cosecha de san valentín. In: Lee, R. (Ed.). Mantenimiento de plantas de rosa, curso de actualización profesional. Centro de investigaciones y Asesorías Agroindustriales Universidad de Bogotá Jorge Tadeo Lozano. Chía. Colombia. 31-48 pp.

Ríos-Ramírez, S. C.; Enríquez-del Valle, J. R.; Rodríguez-Ortiz, G.; Ruíz-Luna, J. and VelascoVelasco, V. A. 2018. In vitro formation of adventitious shoots on caulinary tissue of physiologically contrasting agave angustifolia plants. Emirates J. Food Agr. 30(1):49-56.

Rodríguez, S. M. y Flores, R. V. 2004. Elementos esenciales y beneficiosos en: ferti-riego: tecnologías y programación en agroplasticultura. (Ed.). Programa Cyted Secretaría General. Madrid, España. 25-35 pp.

Silos-Espino, H.; Tovar-Robles, C. L.; González-Cortés, N.; Méndez-Gallegos, S. J. y RosselKipping, D. 2011. Estudio integral del maguey (Agave salmiana): propagacion y valor nutricional. Rev. Salud Pública y Nutrición. 5:75-82.

Uchida, R. 2000. Essential nutrients for plant growth: nutrient functions and deficiency symptoms. In: Silva, A. and Uchida, R. (Ed.) plant nutrient management in hawaii's soils, approaches for tropical and subtropical agriculture. College of Tropical Agriculture and Human Resources, University of Hawaii at Manoa. USA. 31-55 pp.

Vitosh, M. L.; Warncke, D. D. and Lucas, R. E. 1994. Secondary and micronutrients for vegetables and field crops. Michigan State University Extension. USA. 486 p.

Winter, K. and Smith, J. A. C. 1996. An introduction to crassulacean acid metabolism: biochemical principles and ecological diversity. In: Winter, K. and Smith, J. A. C. (Ed.) crassulacean acid metabolism. Springer, Heidelberg. Alemania. 1-13 pp.

Zuñiga, E. L. 2013. Nutrición de Agave tequilana y manejo de los fertilizantes en un sistema de producción intensiva (riego por goteo). Instituto Nacional de Investigaciones Forestales, Agrícolas y Pecuarias (INIFAP). México, DF. 58 p. 